\title{
Cognitive Communicative Abilities in Tinnitus Patients with Normal Hearing
}

\author{
Original \\ Article \\ Naema Ismail', Amani Shalaby'2, Soha Hamada ${ }^{3}$ Heba Abulfadle ${ }^{3}$ \\ ${ }^{1}$ Audiology Department, Faculty of Medicine, Al-Azhar University for Girls, Egypt \\ ${ }^{2}$ Audiology Department, Faculty of Medicine, Ain Shams University, Egypt \\ ${ }^{3}$ Audiovestibular Department, Hearing and Speech Institute, Egypt
}

\begin{abstract}
Background: Tinnitus is a bothersome complain, some studies show that 13-18 percent of the people suffer from tinnitus, almost 5 percent of which, have chronic or bothersome tinnitus. Chronic tinnitus may be accompanied by anxiety, depression, insomnia, and impairment in cognitive functions such as memory and attention.

The Aim of this Study: To compare the auditory memory and auditory attention between two groups with and without tinnitus all of them have normal hearing.

Methods: This study were performed on 30 tinnitus patients (10 men and 20 women) aged from 20 to 50 years old and 30 healthy volunteers ( 12 men and 18 women) aged from 21 to 50 years old with normal hearing thresholds. Pure tone audiometry (PTA), auditory continuous performance test (ACPT), Memory for recognition (MR), memory for content (MC) and memory for sequence (MS) were employed for all participants. Tinnitus evaluation and tinnitus handicap inventory questionnaire (THI) was done for the study group only.

Results: Comparing the results of ACPT between study and control groups show no statistically significant difference. While comparing the results of memory battery test between both groups were statistically highly significant.

Conclusion: Tinnitus decreased the memory battery test scores at the study group in comparison with the control group. Auditory continuous performance test (ACPT) results show no difference between study and control group. Duration of tinnitus affect significantly ACPT results. Bilateral tinnitus affects memory for sequence results. While loudness decreased results of memory for recognition.
\end{abstract}

Key Words: Auditory attention, auditory memory, cognitive abilities.

Received: $19^{\text {th }}$ May 2019, Accepted: $12^{\text {th }}$ December 2019

Corresponding Author: Soha Hamada, M.D., Audiovestibular Department, Hearing and Speech Institute, Egypt, Tel.: +20 1005684518, E-mail: sohahamada@hotmail.com

ISSN: 2090-0740, March 2020 Vol. 21, No. 1.

\section{INTRODUCTION}

Tinnitus is the perception of a sound that has no external source. Some of the more common sounds reported are: ringing, humming, buzzing, and cricket-like. Tinnitus is not a 'phantom sound'. There is real neural activity in brain that are heard as tinnitus ${ }^{[1]}$.

It can also be a combination of sounds, and for many, the sound of their tinnitus actually changes. It can be constant or intermittent and is heard in one ear, both ears or in the head.

The neurophysiologic model explains the severity of tinnitus to be a conditioned response focusing on the network of neural activity in the auditory system, the sympathetic and parasympathetic autonomic nervous systems and the limbic system. Some of the psychological disorders associated with tinnitus are anxiety, depressive disorders, hysteria, insomnia, anger, fear and despair ${ }^{[2]}$.
Researchers have found associations between tinnitus and anxiety disorders and depression ${ }^{[3]}$. Patients with tinnitus and comorbid anxiety and depressive disorder often present with exaggerated symptoms or non-tolerance of tinnitus.

Cognitive-communication disorders are communication impairments resulting from underlying cognitive deficits due to neurological impairment. These are difficulties in communicative competence (listening, speaking, reading, writing, conversation and social interaction) that result from underlying cognitive impairments (attention, memory, organization, information processing, problem solving, and executive functions). When tinnitus is chronic it causes stress on the patient which affect his/her daily activities.

Stress has a significant effect on memory formation and learning. In response to stressful situations, the brain releases hormones and neurotransmitters (ex. glucocorticoids and 
catecholamine) which affect memory encoding processes in the hippocampus. Behavioral research on animals shows that chronic stress produces adrenal hormones which impact the hippocampal structure in the brains of rats ${ }^{[4]}$. Schwabe and Wolf demonstrate how learning under stress also decreases memory recall in humans ${ }^{[5]}$.

Therefore, the more stressful situations that someone encounters, the more susceptible they are to memory loss later on.

Attention is the behavioral and cognitive process of selectively concentrating on a discrete aspect of information, whether deemed subjective or objective, while ignoring other perceivable information ${ }^{[6]}$. Attention is a very basic function that often is a precursor to all other neurological/cognitive functions ${ }^{[7]}$. The presence of tinnitus may automatically direct attention towards the tinnitus ear, which in turn may compromise the function of the involuntary attention system ${ }^{[8]}$.

The cognitive disruption caused by tinnitus might serve as a starting point for later conditioned emotional reactions to tinnitus. Basically, the idea was that tinnitus has an interfering effect on cognitive function, and that this effect is noted by the person who starts to attend to it. Focus on tinnitus leads to less input of other conflicting sounds, and might even be experienced as an increase in loudness (or contrast) ${ }^{[9]}$. This might occur at all steps including when tinnitus first appears, when it is found to disrupt cognitive function, and when fluctuations are perceived. However, the role of cognition is not restricted to the effects of tinnitus on working memory, and cognition occurs at different levels of information processing. More recently, Zenner and Zalaman ${ }^{[10]}$ outlined a cognitive sensitization model. Based on the previous researches and due to the controversy between cognitive functions and tinnitus, and considering the existing contrasts about the effect of tinnitus on cognitive functions, it sounds highly essential to hold a deeper investigation of the impact of tinnitus on the auditory memory functions and attention. Therefore, this study is designed to study the effect of tinnitus on cognitive communicative disorders.

The aim of this study: Evaluate the effect of tinnitus on auditory memory and auditory attention abilities in tinnitus patients with normal hearing and compare them with their normal peers.

\section{SUBJECTS AND METHOD}

The study was approved by ethical community of Faculty of medicine (Girls) Al-Azhar University.

\section{Subjects}

The present study comprises 60 subjects divided into 2 groups: the study group and the control group.

\section{A - Control Group}

This group consists of 30 subjects selected from patients attending at the Hearing and Speech Institute clinics and their relatives. Each subject of this group fulfilled the following criteria .

\section{Inclusion criteria}

- Minimum of 20 and maximum of 50 years old.

- Having normal hearing threshold (mean of threshold less than $25 \mathrm{~dB}$ from $250-8000 \mathrm{~Hz}$ ).

\section{Exclusion criteria}

- No history of ear disease.

- No history of neurological disease.

- No history of brain surgery nor seizure.

- No history of drug abuse.

- No history of tinnitus.

\section{B-Study Group}

This group consists of 30 patients randomly selected from patients attending at the Hearing and Speech Institute clinics. The selection criteria of this group were the same as the control group, However, the patients of this group should suffer from tinnitus for at least 1 year whether unilateral or bilateral tinnitus with exclusion of pulsatile tinnitus.

\section{Equipment}

- Two channels audiometer (Interacoustic model AC 40) calibrated according to ANSI (1969) with sound-field testing facility.

- $\quad$ Sound treated room (I.A.C model 1602).

- Middle ear analyzer (Interacoustic model AZ 26).

- Two channel Computer Compact discs connected to the audiometer and was adjusted to deliver recorded speech material through a loud speaker.

\section{Method}

All subjects were submitted to the following procedures

1. Full history taking.

2. Otological examination.

3. Basic audiological evaluation in the form of:

- Pure tone audiometry: Air conduction thresholds will be tested at the following frequencies $0.25,0.5,1,2,4,8 \mathrm{KHz}$, bone conduction thresholds at the following frequencies $0.5,1,2,4 \mathrm{KHz}^{[11]}$.

- Speech Audiometry: This includes speech reception threshold testing (SRT) using Arabic spondee words ${ }^{[12]}$ and word discrimination score testing using Arabic Phonetically Balanced (PB) words ${ }^{[13]}$.

- Acoustic Immittance Testing: It includes tympanometry and acoustic reflex threshold measurements. 
4. Tinnitus questionnaire: Arabic version of Tinnitus Handicap Inventory $(\mathrm{THI})^{[14 \& 15] \text { : }}$

The questionnaire consists of 25 items. There are three responses to each question.

- The first response," yes", is awarded four points.

- The second response," sometimes", is awarded two points.

- The third response," no", is awarded zero points.

Accordingly, total scores range between zero and 100 points. The final total score was calculated with higher scores representing greater perceived handicap. So, according to THI scoring, patients were are classified into the following:

$$
\begin{aligned}
& \text { - Grade } 1 \text {-slight (THI scoring } 0 \text { to } 16 \text { ). } \\
& \text { - Grade } 2 \text {-mild (THI scoring } 18 \text { to } 36 \text { ). } \\
& \text { - Grade } 3 \text {-moderate (THI scoring } 38 \text { to } 56 \text { ). } \\
& \text { - Grade } 4 \text {-severe (THI scoring } 58 \text { to } 76 \text { ). } \\
& \text { - Grade } 5 \text {-catastrophic (THI scoring } 78 \text { to 100). }
\end{aligned}
$$

5. Measurement of psychoacoustic characteristics of tinnitus:

The basic test protocol involved measurement of 2 tinnitus parameters: (1) pitch, (2) loudness match to external tones.

\section{1-Pitch matching ${ }^{[16]}$}

Pitch is a measurement of tone frequency; the pitch matching is performed by using the 2 alternative forced procedures. Vernon and Fenwick suggested this method as the most reliable for pitch matching ${ }^{[17]}$. Two tones are presented in an alternating manner. The patient is forced to choose which of the 2 tones is more closely matches the pitch of his tinnitus. This continues until pitch matching is made.

\section{2- Loudness matching}

Involved choosing from progressively increasing intensities at $1000 \mathrm{~Hz}$ or the matching frequency until a match is found. Recorded in decibel sensation level (SL, dB above threshold).

6. Psychophysical tests: Four tests were used for the assessment of the memory and attention:

\section{1-Auditory continuous performance test (ACPT)}

This test consisted of nineteen monosyllabic simple Arabic words. These words were repeated many times to form long list of ninety-six Arabic monosyllabic words for the ACPT. The Dog (كلب) was considered the target word and was repeated 20 times. For scoring, incorrect responses were subtracted from 20 then multiplied by 5 .

\section{2-Recognition memory (MR)}

Five lists of Arabic bisyllabic words. Each list consisted of 11 twice repeated words, to form a list of 22 bisyllabic words. Words in each test were belonged to one topic or idea as they are telling a simple story. The scoring of this test is done by counting the number of correct answers at each list and take the mean score of the five lists to be the actual score.

\section{3-Memory for content test (MC)}

Two groups of lists (A) and (B) are present. each group consisted of eight lists of monosyllabic simple Arabic words. The first list comprised two words, the number of words was increased so as to reach the nine words in the eighth list. The scoring of this test is by taking the highest two consistent results of the subject to be the main score. If the score at the two lists is different, we calculate the mean of the two lists score.

\section{4- Memory for sequence test (MS)}

Two groups of lists (A) and (B) are present. Each group consisted of seven lists of monosyllabic simple Arabic words. The first list comprised two words, the number of words was increased so as to reach the eight words in the last list. The respond considered wrong if the subject couldn't recall any word in the list in order (all or none rule). If the score at group A and B of the same list is different, we take the highest score.

All these tests were done while the subject was sitting on chair in the middle of sound treated room .The sound was delivered by 2 loudspeakers at $45^{\circ}$ azimuths with a 50 dB SL presentation level (ref. SRT) $)^{[18]}$.

Correlation between auditory continuous performance test (ACPT), Memory for recognition (MR), Memory for content (MC), Memory for sequence (MS) tests with tinnitus investigations was also studied in this research.

\section{RESULTS}

In this study sixty subjects were included divided into two groups, the control and study groups. They were matched as regards of age and gender.

The tinnitus patients group (the study group) included 30 patients, aging from 20 to 50 years old (mean of 34.43 and standard deviation of 9.50) (10 men and 20 women) . The control group included 30 healthy individuals, aging from 21 to 50 years old (mean of 34.50 and standard deviation of 7.56) (12 men and 18 women). There was no significant difference between the two groups in terms of sex, age $(p>0.05)$. In addition, $60 \%$ of patients were in grade 3 (moderate) as regards THI (Table 1). The mean of tinnitus handicap Inventory score was $(44.07 \%)$ (Table 2$)$. Most of the patients suffers from tinnitus had tinnitus pitch at $1000 \mathrm{~Hz}$ (about 26\%), 18\% had tinnitus pitch at $2000 \mathrm{~Hz}$ , $16.66 \%$ at 250 and $500 \mathrm{~Hz}, 6.66 \%$ at 750,1500 and 4000 $\mathrm{Hz}$, and zero $\%$ at $8000 \mathrm{~Hz}$. Bilateral tinnitus was met at 30 $\%$ of subjects $(n=9)$. In subjects with unilateral tinnitus left sided tinnitus was more prevalent than right sided tinnitus (Table 2). 
Table 1: Distribution of tinnitus patients scores according to Tinnitus Handicap Inventory Questionnaire (THI) Arabic version

\begin{tabular}{lcc}
\hline \multirow{2}{*}{ THI } & \multicolumn{2}{c}{ Tinnitus patients } \\
\cline { 2 - 3 } & No & $\%$ \\
\hline Grade 1 (slight) & 1 & $3.33 \%$ \\
Grade2 (mild) & 6 & $20 \%$ \\
Grade 3 (moderate) & 18 & $60 \%$ \\
Grade 4 (severe) & 5 & $16.66 \%$ \\
Grade 5 (catastrophic) & Zero & Zero $\%$ \\
Total & 30 & $100 \%$ \\
\hline
\end{tabular}

Shows that high percentage of tinnitus patients scores at THI is at grade 3 (moderate).

Table 2: Descriptive analysis of tinnitus pitch matching, loudness matching, laterality, duration and Tinnitus Handicap Inventory Questionnaire (THI) results in study group

\begin{tabular}{ccccc}
\hline & & & $\begin{array}{c}\text { SD } \% \\
\text { interquartile range }\end{array}$ \\
\hline \multicolumn{2}{c}{ Tinnitus pitch } & Mean & 1000.0 & $(500-4000)$ \\
Laterality & Unilateral & No & 21 & $70.0 \%$ \\
& Bilateral & No & 9 & $30.0 \%$ \\
Unilateral & RT & No & 10 & $47.6 \%$ \\
ear & Lt & No & 11 & $52.4 \%$ \\
Loudness (Higher) & Median & 26.8 & 9.8 \\
Loudness (Lower) & Median & 21.83 & 11.02 \\
Duration by months & Median & 28 & 17 \\
Questionnaire by $\%$ & Mean & 44.07 & 12.28 \\
\hline
\end{tabular}

Shows Bilateral tinnitus was met at $30 \%$ of subjects $(n=9)$. In subjects with unilateral tinnitus left sided tinnitus was more prevalent than right sided tinnitus.

All subjects at this study has pure tone threshold in both ears equal to or better than $15 \mathrm{~dB}$ HL at all tested frequencies $(250 \mathrm{~Hz}$ through $8000 \mathrm{~Hz})$. This indicates normal peripheral hearing. Speech audiometry results revealed that speech reception threshold (SRT) were in agreement with the pure tone thresholds. The results showed excellent word discrimination scores for all the subjects. (Table 3) shows no significance difference at ACPT\% between study and control groups. There is a statistical significance reduction in MR, MC, MS scores of study group in relation to the control group as shown in (Table 4). As regards correlation with tinnitus pitch matching, tinnitus loudness matching, THI questionnaire and tinnitus duration, (Table 5) shows significant relation between ACPT\% and tinnitus duration. (Table 6) shows significant relation of MR with loudness (that includes loudness at lesser affected ear of bilateral tinnitus). However, there is no significant relation with MC and MS respectively.

Table 3: Comparison analysis between study and control groups for auditory continuous performance test (ACPT)

\begin{tabular}{|c|c|c|c|c|}
\hline & \multicolumn{2}{|c|}{ Group } & \multirow{2}{*}{\multicolumn{2}{|c|}{$\begin{array}{l}\mathrm{T} \text { - test of } \\
\text { significance }\end{array}$}} \\
\hline & Study & Control & & \\
\hline & Mean \pm SD \% & Mean \pm SD \% & P-Value & Sig. \\
\hline АCPT by $\%$ & $99.33 \pm 2.17$ & $100.00 \pm 0.00$ & 0.103 & NS \\
\hline
\end{tabular}

(C) Chi-Square test of significance.

Shows no statistically significance difference at ACPT\% between study and control groups.

Table 4: Comparison analysis between study and control group at Memory for recognition(MR) test scores, Memory for content (MC) test scores and Memory for sequence (MS) test scores

\begin{tabular}{lcccc}
\hline & \multicolumn{2}{c}{ Group } & \multicolumn{2}{c}{$\begin{array}{c}\text { T-test of } \\
\text { significance }\end{array}$} \\
\cline { 2 - 5 } & Study & Control & \multicolumn{2}{c}{} \\
\cline { 2 - 5 } & Mean \pm SD $\%$ & Mean \pm SD $\%$ & P-Value & Sig. \\
MR & $8.6 \pm 1.3$ & $10.1 \pm 0.5$ & $<0.001$ & HS \\
MC & $4.3 \pm 0.8$ & $6.3 \pm 1.3$ & $<0.001$ & HS \\
MS & $3.4 \pm 0.7$ & $5.2 \pm 1.2$ & $<0.001$ & HS \\
\hline
\end{tabular}

Shows statistical significance reduction in MR, MC, MR scores of study group in relation to the control group.

Table 5: Correlation between auditory continuous performance test (ACPT) and tinnitus investigations

\begin{tabular}{ccccccc}
\hline & & Tinnitus pitch & Loudness (Higher) & Loudness (Lower) & Duration & Questionnaire \\
\hline & Pearson Correlation & 0.187 & -0.103 & 0.125 & -0.386 & -0.050 \\
ACPT by \% & P-Value & 0.324 & 0.589 & 0.511 & 0.035 & 0.793 \\
& Sig. & NS & NS & NS & S & NS \\
\hline
\end{tabular}

(S) Spearman rho correlation test.

Correlation between ACPT\% and tinnitus pitch matching, tinnitus loudness matching, THI questionnaire and tinnitus duration shows significant relation between ACPT\% and tinnitus duration. 
Table 6: Correlation between Memory for recognition (MR), Memory for content (MC) and Memory for sequence (MS) and tinnitus investigations

\begin{tabular}{|c|c|c|c|c|c|c|}
\hline & & Tinnitus pitch & Loudness (Higher) & Loudness (Lower) & Duration & Questionnaire \\
\hline \multirow{3}{*}{ MR } & Pearson Correlation & 0.323 & 0.275 & 0.526 & -0.051 & -0.163 \\
\hline & P-Value & 0.082 & 0.141 & 0.003 & 0.789 & 0.390 \\
\hline & Sig. & NS & $\mathrm{NS}$ & $\mathrm{S}$ & NS & NS \\
\hline \multirow{3}{*}{$\mathrm{MC}$} & Pearson Correlation & -0.052 & -0.016 & 0.316 & -0.112 & -0.128 \\
\hline & P-Value & 0.784 & 0.935 & 0.089 & 0.557 & 0.499 \\
\hline & Sig. & NS & $\mathrm{NS}$ & NS & NS & NS \\
\hline \multirow{3}{*}{ MS } & Pearson Correlation & -0.016 & -0.131 & 0.272 & 0.002 & -0.220 \\
\hline & P-Value & 0.935 & 0.489 & 0.146 & 0.993 & 0.242 \\
\hline & Sig. & NS & NS & NS & NS & NS \\
\hline
\end{tabular}

(S) Spearman rho correlation test.

Correlation between MR and tinnitus pitch matching, tinnitus loudness matching, THI questionnaire and tinnitus duration shows significant relation with loudness (that includes loudness at lesser affected ear of bilateral tinnitus).However, correlation between MC and MS with tinnitus pitch matching, tinnitus loudness matching, THI questionnaire and tinnitus duration shows no significant relation.

\section{DISCUSSION}

Coexistence of various types of cognitive dysfunction result in decreased performance on several tasks that require memory and attention ${ }^{[19]}$. Some models of tinnitus pathophysiology suggest that networks associated with attention, memory, distress and multisensory experience are involved in tinnitus perception.

Not all tinnitus patients suffer from hearing loss as it was observed that $30 \%$ of patients has hearing loss suffer from tinnitus, on the other hand , $15 \%$ of chronic tinnitus patient has normal hearing ${ }^{[20]}$.

In order to know the real effect of tinnitus on memory and attention we examined patients with normal hearing but complaining from chronic tinnitus.

Auditory continuous performance test (ACPT) was used to evaluate the sustained attention ability. Sustained attention means ability to listen over prolonged period of time. In this study the results of comparison between study and control group for ACPT indicates that attention span of tinnitus patient is not affected by the presence of the tinnitus itself. These results matched with findings of Hallam et al., (2004) who conducted a study to see the effect of severe tinnitus on attention in which there was no difference between study and control group ${ }^{[21]}$.

Results of comparison of auditory memory test battery between study and control group show better performance at control group that was highly statistically significant indicating that tinnitus affect memory at tinnitus patient. The results correlates with the statement that" up to $70 \%$ of tinnitus patients do report having difficulties with concentration $^{[22]}$, this being the third highest reported difficulty associated with tinnitus. Also, it is consistent with the results of the study of Advani ${ }^{[23]}$ who studied the effect of tinnitus on working memory and reported impaired performance in the single and dual working memory tasks tinnitus group compared to controls and attributed this to be related to emotional distress.
Memory for sequence test (MS) results were statistically affected in patients who suffered from binaural tinnitus in comparison with monaural tinnitus affection. Although most researches state the impact of chronic tinnitus on the cognitive functions. As, the idea was that tinnitus has an interfering effect on cognitive function and that this effect is noted by the person. Who starts to focus on tinnitus that leads to less input of other conflicting sounds ${ }^{[10]}$. Others researchers reported no interference of tinnitus in the behavioral-cognitive functions (attention and memory $)^{[24,25,26]}$.

The effect of different variables, namely: duration, pitch and loudness and THI questionnaire of the tinnitus on memory and attention tests were studied in this research using the analysis of variance technique.

This research showed that the duration of the tinnitus significantly affects the auditory continuous performance test., the longer the duration of the tinnitus the lower the attention span of the patient. Similar finding were reported by (Mohamad et al., ${ }^{[27]}$ who suggest that the duration of tinnitus may interfere with cognitive processing especially the performance in digit span, verbal comprehension, mental balance, attention,concentration, and immediate recall. Heeren et al., also reported that the degree of the executive control impairment of the tinnitus patient was significantly correlated with duration of tinnitus ${ }^{[28]}$.

Loudness of the tinnitus (including lesser affected ear at bilateral tinnitus) statistically affects memory for recognition results with no effect of different variable on memory for content nor memory for sequence as shown in this study.

Finally, cognitive communicative abilities should be evaluated in each tinnitus patient to avoid the impact of tinnitus on his performance and provides these patients with appropriate rehabilitation. 


\section{CONCLUSION}

The influence of tinnitus on cognitive abilities were evaluated in this study. The findings revealed that tinnitus decreases the memory battery test scores at the study group in comparison with the control group. However, no difference were detected as regards Auditory continuous performance test results between both groups. Attention span affected by duration of tinnitus, the more the duration of the tinnitus the less attention span of the patient. Also, I $t$ was found that bilateral tinnitus decreases memory for sequence scores. However, Memory for recognition results decreased by loudness.

\section{CONFLICT OF INTEREST}

There are no conflict of interest

\section{REFERENCES}

1. Non dahl, D.M., Cruickshanks, K.J., Huang, G.H., Klein, B.E., Klein, R., Javier Nieto, F. and Tweed, T.S., (2011): Tinnitus and its risk factors in the Beaver Dam offspring study. International journal of audiology, 50(5), pp.313-320

2. Jastreboff, P.J. and Hazell, J.W., (2008): Tinnitus retraining therapy: Implementing the neurophysiological model. Cambridge University Press.

3. Krog, N.H., Engdahl, B.O. and Tambs, K., (2010) :The association between tinnitus and mental health in a general population sample: results from the HUNT Study. Journal of psychosomatic research, 69(3), pp.289-298.

4. Conrad, C.D., (2010) : A critical review of chronic stress effects on spatial learning and memory. Progress in Neuro-Psychopharmacology and Biological Psychiatry, 34(5), pp.742-755.

5. Schwabe, L. and Wolf, O.T., (2010) : Learning under stress impairs memory formation. Neurobiology of learning and memory, 93(2), pp.183-188.

6. Anderson, J.R., (2004) : Cognitive psychology and its implications. Macmillan.

7. McKay Moore Sohlberg \&Catherine A. Mateer:(1987): Effectiveness of an attention-training program ,Journal of Clinical and Experimental Neuropsychology Volume 9, Issue 2.

8. Cuny, C., Norena, A., El Massioui, F. and ChéryCroze, S., 2004. Reduced attention shift in response to auditory changes in subjects with tinnitus. Audiology and Neurotology, 9(5), pp.294-302.

9. De Houwer, J., Thomas, S. and Baeyens, F., 2001. Association learning of likes and dislikes: A review of 25 years of research on human evaluative conditioning. Psychological bulletin, 127(6), p.853.
10. Zenner, H.P. and Zalaman, I.M., 2004 Cognitive tinnitus sensitization: behavioral and neurophysiological aspects of tinnitus centralization. Acta oto-laryngologica, 124(4), pp.436-439.

11. ASHA (2005): Guidelines for Manual Pure-Tone Threshold Audiometry .

12. Soliman, A. Fathalla, M. Shehata (1985): Development of Arabic staggered spondee words (SSW) test Proceedings of 8th Ain Shams Med Congress, vol. 12, Ain Shams University ), pp. 1220-1246

13. Soliman S.( 1976): Speech discrimination audiometry using Arabic phontically balanced words. Ain Shams Med J; 27: 27-30.

14. Newman, C.W., Jacobson, G.P. and Spitzer, J.B., (1996): Development of the tinnitus handicap inventory. Archives of Otolaryngology-Head \& Neck Surgery, 122(2), pp.143-148.

15. Kamal et al.,(2005) :Tinnitus questionnaire: Tinnitus Handicap Inventory (THI) Questionnaire Arabic version .

16. Segal, N. T., M. Puterman, M. Shkolnik, A. Niv, D. Kaplan, A. Kochva, and M. Kraus. (2007): "The Role of Tinnitus Evaluation Tests in Differentiating Functional Versus Organic Tinnitus.” OtolaryngologyHead and Neck Surgery: Official Journal of American Academy of Otolaryngology-Head and Neck Surgery 137 (5): 772-775.

17. Vernon, J. and Fenwick, J.,( 1984) : Identification of tinnitus: a plea for standardization. The Journal of Laryngology \& Otology, 98(S9), pp.45-53.

18. Abumoussa, H., Tawfik, S., El-Danasory, E. and AbdelMaksoud, A., (2002) : Assessment of attention and memory in scholastic underachievers: psychophysical and electrophysiological studies. Unpublished MD thesis.

19. Rossiter, S., Stevens, C. and Walker, G., 2006. Tinnitus and its effect on working memory and attention. Journal of speech, language, and hearing research, 49(1), pp.150-160.

20. Eggermont, J.J. and Roberts, L.E.,( 2015): Tinnitus: animal models and findings in humans. Cell and tissue research, 361(1), pp.311-336.

21. Hallam, R.S., McKenna, L. and Shurlock, L.,(2004): Tinnitus impairs cognitive efficiency. International journal of audiology, 43(4), pp.218-226.

22. Andersson, 2009: Behav Res Ther. (2009) : Mar;47(3):175-80. doi: 10.1016/j.brat.2009.01.010. Epub 2009 Feb 20.Using the Internet to provide cognitive behaviour therapy.

23. Advani, J., (2011).:The effects of tinnitus on working memory (Doctoral dissertation, University of London). 
24. Acrani IO, Pereira LD. (2010): Temporal resolution and selective attention of individuals with tinnitus. Pro Fono.;22(3):233-8.

25. Husain FT, Pajor NM, Smith JF, Kim HJ, Rudy S, Zalewski C, et al. (2011): Discrimination task reveals differences in neural bases of tinnitus and hearing impairment. PLoS One.;6(10): e26639.

26. Husain, F.T., Akrofi, K., Carpenter-Thompson, J.R. and Schmidt, S.A., (2015) : Alterations to the attention system in adults with tinnitus are modality specific. brain research, 1620, pp.81-97.
27. Mohamad, N., Hoare, D.J. and Hall, D.A., (2016); The consequences of tinnitus and tinnitus severity on cognition: a review of the behavioural evidence. Hearing research, 332, pp.199-209.

28. Heeren, A., Maurage, P., Perrot, H., De Volder, A., Renier, L., Araneda, R., Lacroix, E., Decat, M., Deggouj, N. and Philippot, P.,( 2014) :Tinnitus specifically alters the top-down executive control subcomponent of attention: evidence from the attention network task. Behavioural brain research, 269, pp.147-154. 